\title{
Angiotensin II type 1 receptor-independent beneficial effects of telmisartan on dietary-induced obesity, insulin resistance and fatty liver in mice
}

\author{
X. Rong $\cdot$ Y. Li $\cdot$ K. Ebihara $\cdot$ M. Zhao $\cdot$ J. Naowaboot $~$ \\ T. Kusakabe • K. Kuwahara • M. Murray • K. Nakao
}

Received: 4 January 2010/Accepted: 4 March 2010 /Published online: 15 April 2010

(C) Springer-Verlag 2010

\begin{abstract}
Aims/hypothesis Evidence suggests that telmisartan, an angiotensin II type 1 receptor (AT1) blocker and peroxisome proliferator-activated receptor- $\gamma$ partial agonist, has beneficial actions that limit development of the metabolic syndrome and diabetes. However, the role played by AT1 inhibition in metabolic effects elicited by telmisartan remains uncertain. Here we isolated the metabolic effects of telmisartan from AT1 antagonism.

Methods Male Atla (also known as Agtrla)-deficient mice were fed a standard diet or $60 \%$ high-fat diet; those on high-fat diet were co-administered telmisartan $\left(3 \mathrm{mg} \mathrm{kg}^{-1}\right.$ day $^{-1}$ by oral gavage) or vehicle for 12 weeks.

Results In At1a-null mice, telmisartan prevented high-fatdiet-induced increases in (1) body weight, epididymal and inguinal white adipose tissue weight, adipocyte size and plasma leptin concentration; (2) plasma glucose and insulin concentrations and HOMA index; and (3) liver weight and triacylglycerol content. Insulin tolerance testing also indicated that telmisartan improved the high-fat-diet-induced reduction of glucose-lowering by insulin.
\end{abstract}

Electronic supplementary material The online version of this article (doi:10.1007/s00125-010-1744-6) contains supplementary material, which is available to authorised users.

X. Rong $\cdot$ Y. Li $(\bowtie) \cdot$ K. Ebihara $\cdot$ M. Zhao $\cdot$ J. Naowaboot $\cdot$

T. Kusakabe $\cdot$ K. Kuwahara $\cdot$ K. Nakao

Department of Medicine and Clinical Science,

Kyoto University Graduate School of Medicine,

Kyoto 606-8507, Japan

e-mail: yuhao@kuhp.kyoto-u.ac.jp

M. Murray

Laboratory of Pharmacogenomics, Faculty of Pharmacy,

The University of Sydney,

Sydney, NSW 2006, Australia
Conclusions/interpretation The present findings demonstrate beneficial, AT1-independent effects of the AT1 blocker telmisartan on dietary-induced obesity, insulin resistance and fatty liver in animals.

Keywords Angiotensin II type 1 receptor - Fatty liver. Insulin resistance $\cdot$ Obesity $\cdot$ Telmisartan
Abbreviations
ARB Angiotensin II type 1 receptor blocker
AT1 Angiotensin II type 1 receptor
eWAT Epididymal white adipose tissue
HFD High-fat diet
ITT Insulin tolerance test
iWAT Inguinal white adipose tissue
KO Knockout
PPAR Peroxisome proliferator-activated receptor

\section{Introduction}

The metabolic syndrome is a cluster of conditions arising from overnutrition and a sedentary lifestyle. Common components of the metabolic syndrome include abdominal obesity, insulin resistance, hypertension and dyslipidaemia. Additional comorbidities may also be present, such as hepatic steatosis leading to non-alcoholic fatty liver disease, proinflammatory and prothrombotic states, and reproductive disorders [1]. Insulin resistance is the key component of the metabolic syndrome. It precedes and predicts the incidence and development of type 2 diabetes and cardiovascular diseases [2].

It has been suggested that activation of the reninangiotensin system is a common feature in patients with 
obesity/the metabolic syndrome [2]. Indeed, blockade of the renin-angiotensin system has been shown in clinical and experimental studies to improve the metabolic syndrome [2]. Most of the established physiological and pathophysiological effects of angiotensin II appear to be mediated through the angiotensin II type 1 receptor (AT1). Angiotensin II type 1 receptor blockers (ARBs) have been used widely in the clinic as antihypertensive agents. More recently, treatment with some ARBs has been found to improve insulin resistance and to protect against the onset and development of type 2 diabetes in insulin-resistant patients with hypertension [2]. Studies in experimental animals seem to support clinical findings [2]. Thus, although the precise mechanisms have yet to be established, the contribution of AT1 signalling and the beneficial effects of AT1 inhibition in the metabolic syndrome are undoubted.

Telmisartan is a well-established ARB. Recently, it was shown to be a partial agonist of the peroxisome proliferatoractivated receptor (PPAR) $\gamma$ in cultured cells [3, 4] and to activate PPAR $\alpha$ in cultured hepatic cells and in liver of wild-type mice fed a high-fat diet (HFD) [5]. Telmisartan has also been reported to improve HFD-induced obesity, insulin resistance and fatty liver in wild-type animals $[4,5]$. However, the extent to which AT1 inhibition contributes to the beneficial effects of telmisartan treatment has not yet been established. The present study tests the role of AT1 inhibition in telmisartan-elicited metabolic effects using HFD-fed Atla (also known as Agtrla)-knockout (KO) mice.

\section{Methods}

Animals, diet and experimental protocol All animal procedures were in accordance with the Principles of Laboratory Animal Care (http:/grants1.nih.gov/grants/olaw/references/ phspol.htm) and were approved by the Animal Ethics Committee, Kyoto University, Japan. Mice were housed in a temperature-controlled facility $\left(21 \pm 1^{\circ} \mathrm{C}, 55 \pm 5 \%\right.$ relative humidity) with a 12-h light/dark cycle (three to four mice per cage).

Male C57BL/6J wild-type mice were purchased from Japan SLC (Shizuoka, Japan). Male Atla-KO mice on a C57BL/6J background were generated by methods that have been described previously [6]. Animals ( 8 weeks of age) were divided into three groups $(n=8-10$ each): (1) standard diet control (standard diet Tel-); (2) HFD control (HFD Tel-); and (3) HFD telmisartan (HFD Tel+) groups. The animals had free access to water and the standard diet (CLEA, Tokyo, Japan) (Tel- group) or HFD (containing $20 \%$ [wt/wt] protein, $20 \%$ [wt $/ \mathrm{wt}$ carbohydrate and $60 \%$ [wt/wt] fat) (D12492; Research Diet, New Brunswick, NJ, USA) (HFD Tel- and HFD Tel+ groups). Animals receiving the HFD were also administered telmisartan (3 $\mathrm{mg} / \mathrm{kg}$ suspended in $5 \%$ (wt/vol.) gum arabic (a gift from Boehringer Ingelheim, Tokyo, Japan) or vehicle by oral gavage once daily (between 11:00 and 12:00 hours) for 12 weeks. Daily $(24 \mathrm{~h})$ food intake was estimated from weekly measurements. Systolic BP was measured by a tailcuff method (MK-2000ST; Muromachi Kikai, Tokyo, Japan) at week 5. Blood samples were collected by retroorbital venous puncture under ether anaesthesia at week 10 in animals in non-fasted and fasted $(12 \mathrm{~h})$ states. Insulin tolerance tests (ITT; $0.75 \mathrm{IU} / \mathrm{kg}$, i.p.; Humulin RInsulin; Eli Lilly, Indianapolis, IN, USA) were conducted after fasting $(6 \mathrm{~h})$ at week 11. After animals were weighed at week 12, blood samples were collected again from non-fasted animals. Immediately thereafter animals were killed by prompt dislocation of the neck vertebra. Epididymal white adipose tissue (eWAT), inguinal white adipose tissue (iWAT) and liver were collected and weighed. Gastrocnemius was also collected. Glucose, triacylglycerol and NEFA concentrations were determined colorimetrically (Wako, Osaka, Japan), and insulin and leptin concentrations were analysed by ELISA (Morinaga, Tokyo, Japan). HOMA was calculated by method that has been described previously [7].

A portion of eWAT or liver was fixed with $10 \%$ formalin (vol./vol.) and embedded in paraffin. Sections $(20 \mu \mathrm{m})$ were cut and stained with haematoxylin and eosin for examination of adipose tissue and liver histology (IX-81; Olympus, Tokyo, Japan). The adipocyte cross-sectional area was measured using an image analysing system (KS 400; Carl Zeiss Vision, Eching, Germany).

Data analysis All results are expressed as means \pm SEM. Data were analysed by one-way ANOVA. If a difference was detected ( $F$ ratio), the Student-Newman-Keuls test was performed to locate the differences between groups. Values of $p<0.05$ were considered to be statistically significant.

\section{Results}

Consistent with previous reports $[4,5]$, we have demonstrated that telmisartan treatment $(3 \mathrm{mg} / \mathrm{kg})$ did not affect food intake (Electronic supplementary material [ESM] Fig. 1b), but substantially attenuated HFD-induced obesity (ESM Fig. 1c-h), insulin resistance (ESM Fig. 2a-e) and lipid overaccumulation in liver and skeletal muscle (ESM Fig. 3c-g) in C57BL/6J wild-type mice. In the present study, systolic BP in Atla-KO mice was $75 \pm 2.5 \mathrm{mmHg}$ (Fig. 1a), which was lower than in wild-type mice (ESM Fig. 1a); telmisartan did not alter systolic BP in At1a-KO mice. 
a

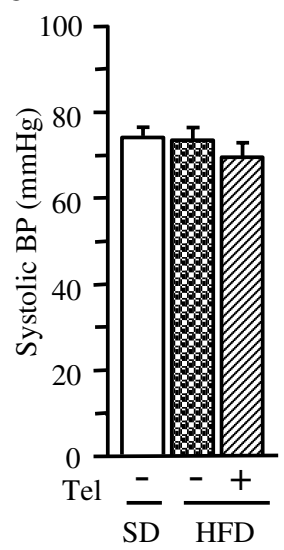

g

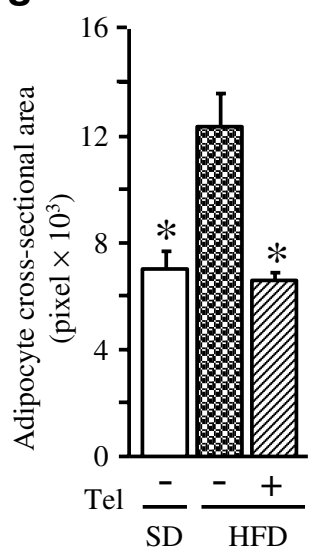

b

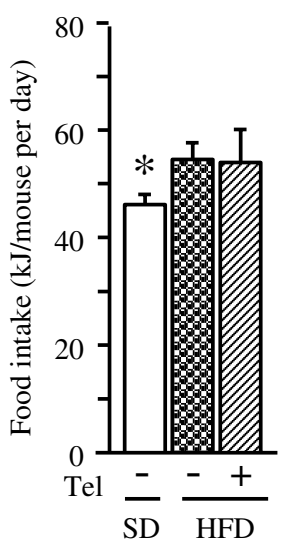

C

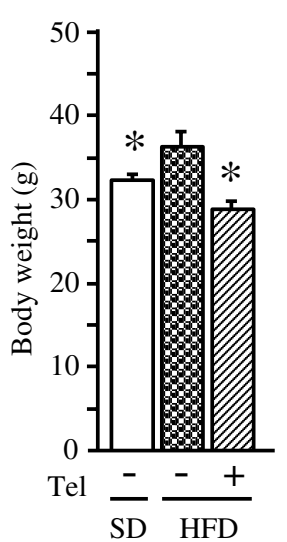

d

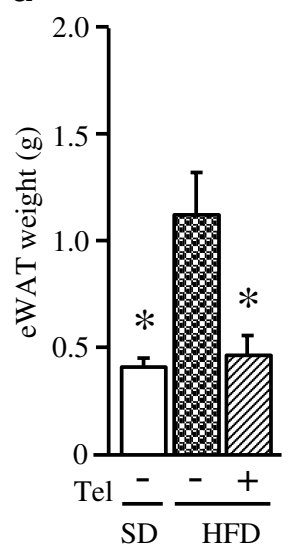

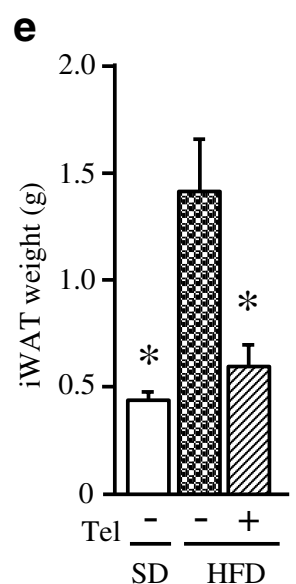

f

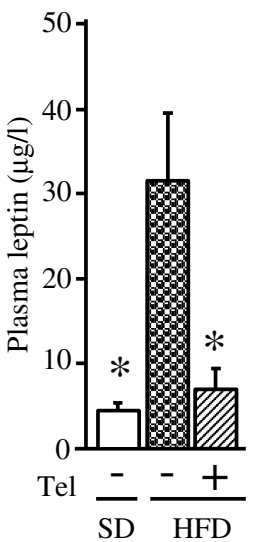

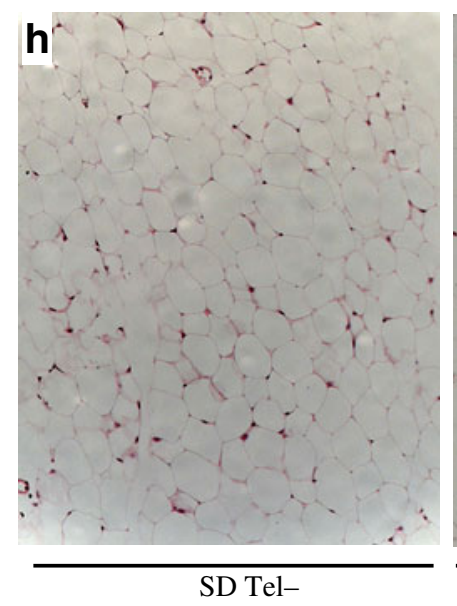

SD Tel-

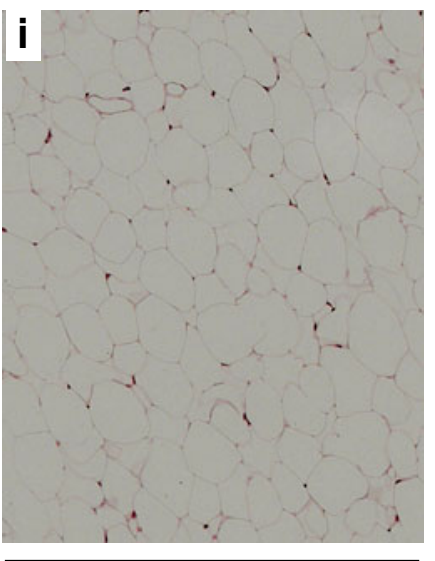

HFD Tel-

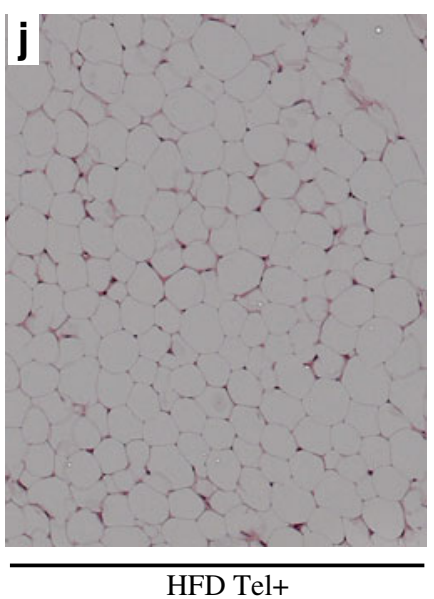

HFD Tel+
Fig. 1 Systolic BP (a), food intake per day (b), body weight (c), eWAT weight (d), iWAT weight (e), plasma leptin concentration (f), adipocyte cross-sectional area (g) and representative images $(\mathbf{h}-\mathbf{j})$ showing histology of eWAT (haematoxylin and eosin staining, magnification $\times 200$ ) in male At1a-KO mice fed standard or HFD for 12 weeks. All values are means $\pm \operatorname{SEM}(n=8-10) ; * p<0.05$ vs HFD control (Tel-). Tel-, vehicle; Tel+, telmisartan $3 \mathrm{mg} / \mathrm{kg}$
HFD feeding significantly increased food intake (Fig. 1b), body weight (Fig. 1c), the weight of eWAT (Fig. 1d) and iWAT (Fig. 1e), adipocyte size (Fig. 1g-i) and plasma leptin concentration (Fig. 1f) in Atla-KO mice. Telmisartan treatment did not affect food intake, but did ameliorate other variables (Fig. 1c-g, j).

In Atla-KO mice, HFD feeding increased the fasted plasma glucose (Fig. 2a) and insulin (Fig. 2b) concentrations, and the HOMA index (Fig. 2c) in comparison with those in standard diet-fed Atla-KO mice. Plasma glucose concentrations after insulin challenge (Fig. 2d) and the glucose AUC during ITT (Fig. 2e) were also increased by HFD treatment. Telmisartan treatment suppressed the HFDinduced increase in all variables.

HFD feeding had no effect on non-fasted and fasted plasma concentrations of triacylglycerol and NEFA (Fig. 2f, $\mathrm{g}$ ), and on liver weight (Fig. 2h), whereas triacylglycerol content in liver and skeletal muscle was increased against that in standard diet-fed At1a-KO mice (Fig. 2i, j).
Telmisartan treatment decreased liver weight and triacylglycerol content in liver and skeletal muscle. Histological evaluation confirmed that fatty infiltration of the liver was decreased by telmisartan (data not shown). In contrast with the lipid-lowering effects observed in HFD-fed wild-type rats [4] and mice (ESM Fig. 3a, b), telmisartan minimally affected non-fasted and fasted plasma triacylglycerol and NEFA concentrations in HFD-fed At1a-KO mice.

\section{Discussion}

The present study demonstrates that long-term treatment with telmisartan in At la-null mice fed a HFD prevents or improves the increase in: (1) body weight, white adipose tissue weight, adipocyte size and plasma leptin concentration; (2) plasma glucose and insulin concentrations, HOMA index, and plasma glucose concentrations and glucose AUC during ITT; and (3) liver weight and triacylglycerol content of the 

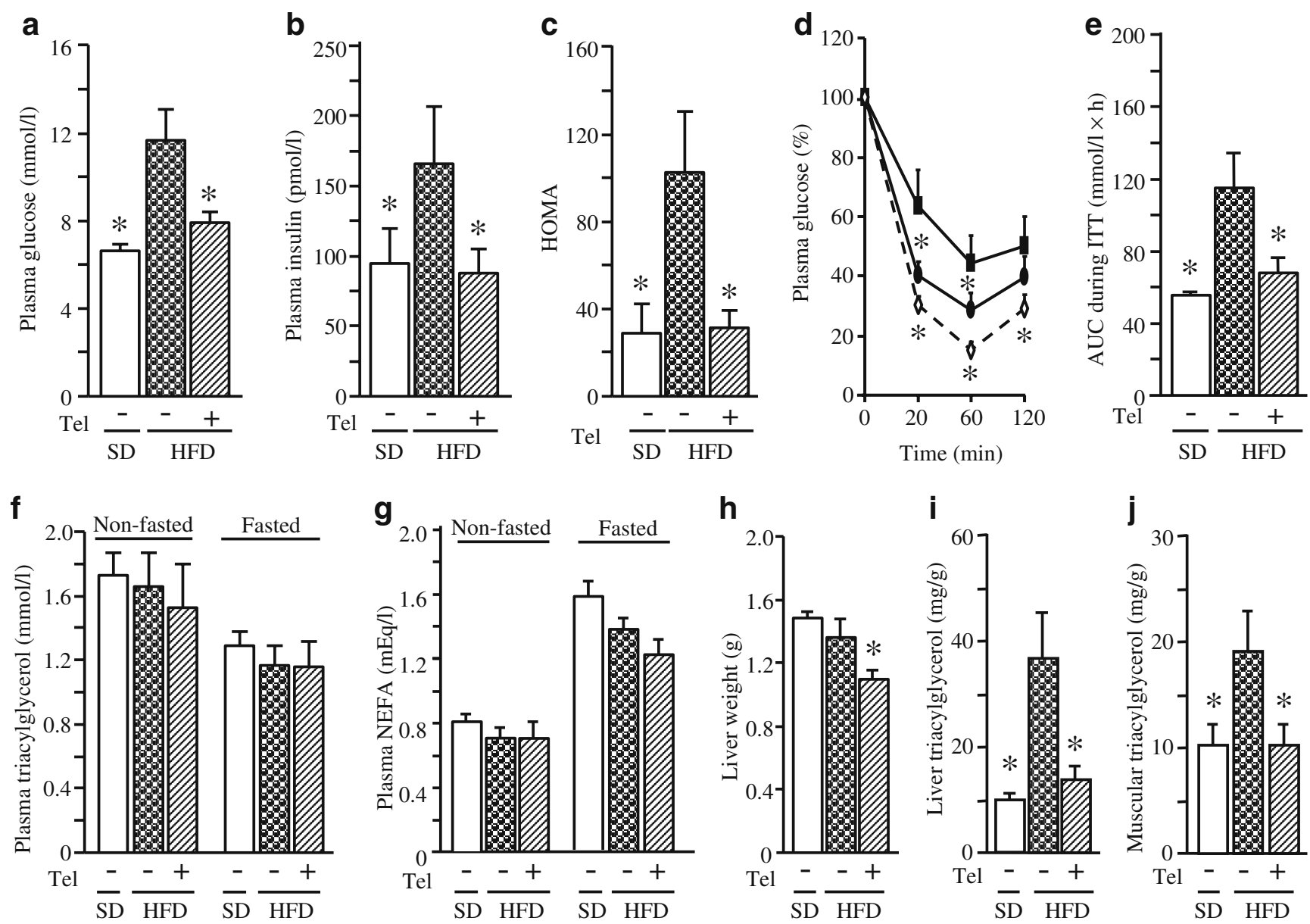

Fig. 2 Fasted (12 h) plasma concentrations of glucose (a) and insulin (b), HOMA index (c), plasma glucose response curve (per cent glucose change) (d), plasma glucose AUC following insulin challenge in ITT (Insulin $0.75 \mathrm{IU} / \mathrm{kg}$, i.p.) (e), non-fasted and fasted plasma triacylglycerol concentrations (f), NEFA concentrations (g), liver weight (h), and

liver and skeletal muscle. The present findings reveal AT1independent improvement of dietary-induced obesity, insulin resistance and fatty liver following telmisartan treatment.

Obesity is a well-established metabolic and cardiovascular risk factor. Recent advances have increased our understanding of the cellular mechanisms whereby adiposity induces adverse local and systemic effects. These include lipid accumulation in adipocytes, induction of endoplasmic reticulum and mitochondrial stress, and insulin resistance. Increased adipose tissue mass, especially in the visceral compartment, represents one of the major risk factors for development of type 2 diabetes [8]. We have recently demonstrated that telmisartan treatment improved insulin resistance and fatty liver in A-ZIP/F-1 transgenic mice lacking adipose tissue [7]. These results suggest that telmisartan may elicit its metabolic effects independently of adipose tissue. In the present study, telmisartan treatment attenuated obesity induced by HFD, a finding accompanied by improvement of insulin resistance in wild-type and triacylglycerol content of liver and skeletal muscle respectively (i, $\mathbf{j})$ in male At1a-KO mice fed a standard diet or HFD. All values are means \pm SEM $(n=8-10) ;{ }^{*} p<0.05$ vs HFD control (Tel-). Tel-, vehicle; Tel+, telmisartan $3 \mathrm{mg} / \mathrm{kg}$; (d) white diamonds, standard diet Tel-; black squares, HFD Tel-; black ovals, HFD Tel+

At1a-KO mice. Thus, the present results suggest that adipose tissue may be an alternative pathway for the insulin-sensitising effect of telmisartan.

On the other hand, an increasing body of evidence indicates that several manifestations of the metabolic syndrome and type 2 diabetes mellitus, including insulin resistance, occur as a result of overaccumulation of lipids in non-adipose tissues, such as liver and skeletal muscle [9]. A decrease in hepatic triacylglycerol pools leads to improved insulin sensitivity [10]. In the present study, HFD-feeding induced excessive lipid accumulation in liver and skeletal muscle, whereas telmisartan treatment ameliorated hepatic steatosis and muscular triacylglycerol deposition in Atladeficient mice. These results imply that attenuation of dietinduced fatty liver by telmisartan is driven by mechanisms that are independent of AT1. Thus, amelioration of lipid overaccumulation in non-adipose tissues may be an important factor associated with improved insulin action in At1a-null mice following treatment with telmisartan. 
In conclusion, the present findings demonstrate for the first time AT1-independent beneficial effects of telmisartan on diet-induced obesity, insulin resistance and fatty liver in mice. Activation of PPAR $\gamma$ might be one potential AT1independent mechanism of action.

Acknowledgements We thank Y. Yamamoto (Kyoto University) for useful discussions during the preparation of this manuscript and $\mathrm{W}$. Aini for his technical assistance during the project.

Duality of interest The authors declare that there is no duality of interest associated with this manuscript.

\section{References}

1. Cornier MA, Dabelea D, Hernandez TL et al (2008) The metabolic syndrome. Endocr Rev 29:777-822

2. Prasad A, Quyyumi AA (2004) Renin-angiotensin system and angiotensin receptor blockers in the metabolic syndrome. Circulation 110:1507-1512
3. Fujimoto M, Masuzaki H, Tanaka T et al (2004) An angiotensin II AT1 receptor antagonist, telmisartan augments glucose uptake and GLUT4 protein expression in 3T3-L1 adipocytes. FEBS Lett 576:492-497

4. Benson SC, Pershadsingh HA, Ho CI et al (2004) Identification of telmisartan as a unique angiotensin II receptor antagonist with selective PPARgamma-modulating activity. Hypertension 43:993-1002

5. Clemenz M, Frost N, Schupp M et al (2008) Liver-specific peroxisome proliferator-activated receptor alpha target gene regulation by the angiotensin type 1 receptor blocker telmisartan. Diabetes 57:1405-1413

6. Li Y, Kishimoto I, Saito Y et al (2002) Guanylyl cyclase-A inhibits angiotensin II type 1A receptor-mediated cardiac remodeling, an endogenous protective mechanism in the heart. Circulation 106:1722-1728

7. Rong X, Li Y, Ebihara K et al (2009) An adipose tissueindependent insulin-sensitizing action of telmisartan: a study in lipodystrophic mice. J Pharmacol Exp Ther 331:1096-1103

8. Bloomgarden ZT (2000) Obesity and diabetes. Diabetes Care 23:1584-1590

9. Unger RH (2002) Lipotoxic diseases. Annu Rev Med 53:319-336

10. Neschen S, Morino K, Hammond LE et al (2005) Prevention of hepatic steatosis and hepatic insulin resistance in mitochondrial acyl-CoA:glycerol-sn-3-phosphate acyltransferase 1 knockout mice. Cell Metab 2:55-65 